\title{
Kinetics Modeling for Slider-Crank Mechanism
}

\author{
Yong H. Chung, Eui K. Ahn, Won K. Hwam, \\ Sang C. Park \\ Department of Industrial Engineering \\ Ajou University \\ Suwon, Korea \\ yongho1230@gmail.com
}

\author{
Jung J. Choi \\ Department of Media \\ Ajou University \\ Suwon, Korea \\ jungju@ajou.ac.kr
}

\begin{abstract}
This paper proposes a procedure to extract the kinetic model from the geometric model of a fixture to reduce the modeling time and effort. Since most of the fixtures in industry follow the same kinetic mechanism, the so-called a slider-crank mechanism; this is a four-axis system of three revolute and one prismatic joint. The prismatic axis of a fixture represents a pneumatic actuator involving a piston and a cylinder. It is very important to identify the prismatic axis from a given geometric model to extract the kinetic model of a fixture. The focus of this paper is to extract a common axis from contact surface between distinguishable links. To do so, we use the concept of the 'Gauss map'.
\end{abstract}

Keywords : kinetic model; geometric model; fixture; slidercrank mechanism, gauss map, common axis

\section{INTRODUCTION}

The competition of manufacturing industry has been changed rapidly due to the enormous improvement of computing power. People want to create much more realistic simulation models for effective and efficient production. Simulation is based on an abstract model of the plant that generates production reports and statistics. The process, however, is time-consuming, and the generated results often do not match the expectations of experienced users or factory historical record. This mismatch places the usability of simulation systems in doubt, and causes hesitation in adopting them. This situation has resulted in the concept of a virtual factory through integrating $3 \mathrm{D}$ visualization, which can be described as a model executing virtual manufacturing processes within a computer simulation[1-3]. A 3D virtual factory is a very useful model for analyzing complex manufacturing systems that cannot be described by analytical or mathematical models. It is necessary to design fixture that consume a major portion of production development time.

A fixture is a device used to locate, hold and support a workpiece during a manufacturing process, as shown in Fig. 1. Fixtures play an important role in shortening production cycle time and ensuring production quality, and thus reducing production cost. Fixture design, fabrication, and testing consume a major portion of production development time. They are required in most automated manufacturing system such as assembly line, which can improve the productivity. However its efficiency depends greatly on fixture design and functionality. Fixture design is a highly experience-based process, which usually requires 10 and more years of manufacturing practice to design quality fixtures. It is also a tedious and time-consuming task. It, however, is crucial to issues of quality, cycle time, and cost of production.

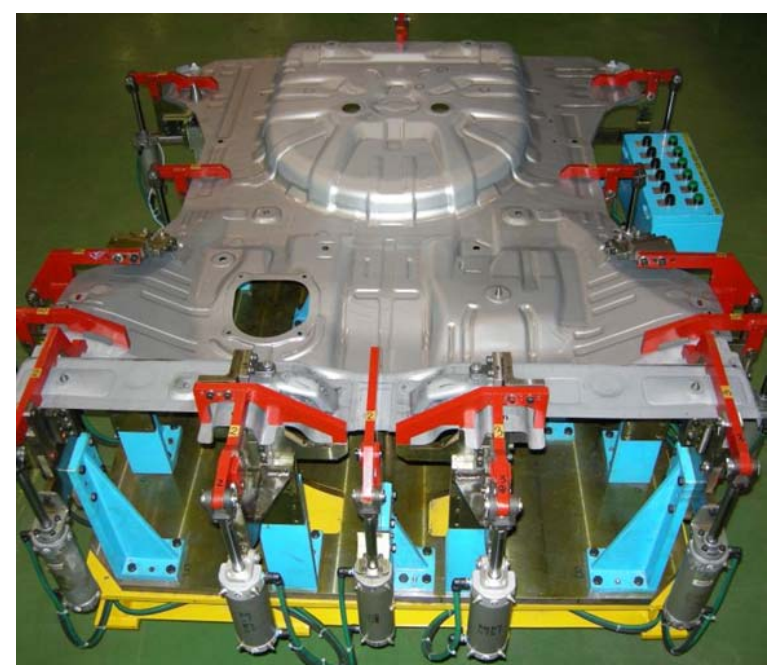

Figure 1. A FIXTURE WITH KINEMATICS

The automation of fixture design process is successfully solved by applying the system for Computer-aided fixture design(CAFD). CAFD with verification has become a means of providing solutions in fixture design. CAFD has emerged as an integration of fixture design knowledge with 3dimensional CAD data (Pehlivan et al. 2008, Mervyn et al. 2008). Motivation of CAFD has been derived from the demand of rapid generation of conceptual and detail fixture designs even in product and production design stages, providing tools for fixture design and process verification, and CAD/CAM integration.

Since many researchers have given a great deal of attention to fixture modeling for a given workpiece, many modeling methodologies have been developed. Asada and By used the Jacobian Matrix to model the fixture-workpiece relationship in 3D space[4]. Chou, Chandru et al. developed a screw theory for fixture analysis and synthesis[5]. The time-variant stability problem was discussed with consideration of fixture force limits and directions[6]. Recently, Mervyn, Kumar et al. developed an evolutionary 
search algorithm that aids a fixture designer by exploring the large number of possible alternatives and suggesting an appropriate design[7]. Choubey, Prakash et al. proposed a genetic algorithm with learning automata algorithm (GALA) for the fixture layout optimization problem[8]. The GALA algorithm is a population based interconnected learning automata algorithm incorporating genetic operators. Although we can find many previous results on optimized fixture design and analysis, few investigations have been reported on the automation of fixture design procedure. This serves as motivation to explore the possibility of finding an appropriate methodology supporting the automation of fixture design, meeting practical requirements of an automotive body assembly line.

This paper deals with automated fixture modeling for automotive body assembly lines. Minho Chang et al proposed an algorithm extracting the kinetic model from the geometric model of a fixture to reduce the fixture modeling time and effort[10]. Its algorithm, however, assumes that geometric model consists of primitives, such as boxes, spheres, and cylinders, and created common axis of each joint by converting each cylindrical feature into a segment tagged with a radius.

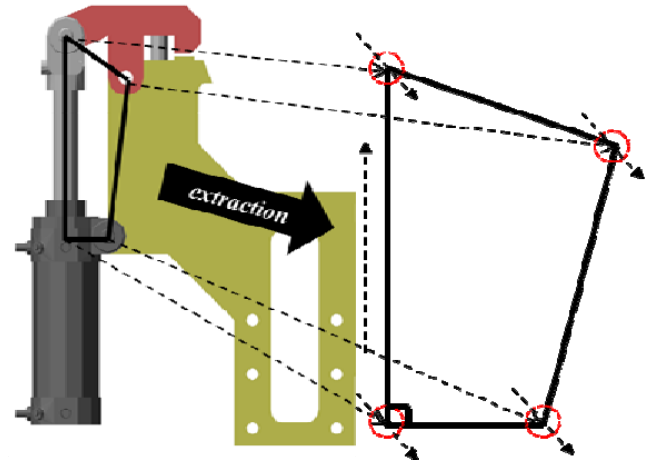

Figure 2. KINETIC MODEL EXTRACTION FROM A GEOMETRIC MODEL.

This paper proposes a procedure to extract the kinetic model from the geometric model of a fixture to reduce the modeling time and effort, as shown in Fig. 2. A fixture model consists of two sub-models, as depicted by Kang, Rong et al: a geometric model and a kinetic model[9]. The geometric model describes the relationship between workpiece displacement and locator displacement. The kinetic model describes the relationship between external forces and workpiece displacement. The focus of the paper is to create a common axis of each joint to generate the kinetic model of a fixture from a given geometric model.

In the remainder of this paper, Section 2 presents the overall approach to the automatic generation of a kinetic model from a given geometric model. After Section 3 details the proposed algorithm, Section 4 presents concluding remarks.

\section{APPROACH TO THE AUTOMATIC GENERATION OF A} KINETIC MODEL

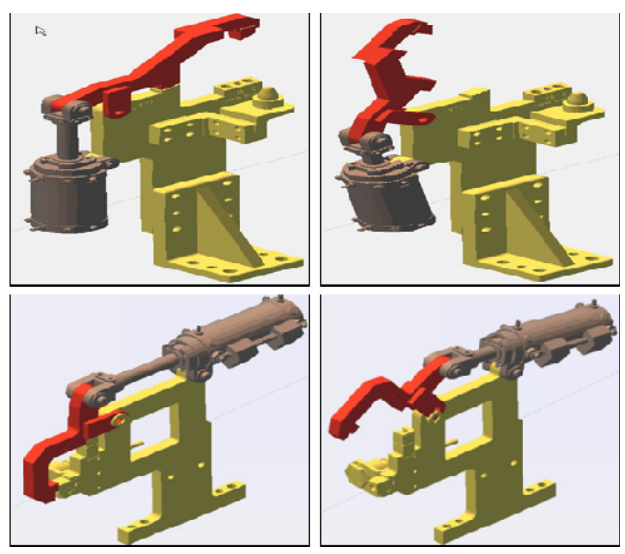

Figure 3. VARIOUS FIXTURES USED IN AUTOMOTIVE BODY ASSEMBLY LINES.

Since an automotive body assembly line is one of highly automated, it is very important to have an efficient design methodology for fixtures. There are various types of fixtures used in automotive body assembly, as shown in Fig. 3. Although the geometric models of those fixtures look very different, their kinetic models follow the same mechanism, the so-called slider-crank mechanism. Fig. 4 shows that slider-crank mechanism consists of four bodies linked with three revolute joints and one sliding or prismatic joint. It is used to change circular into reciprocating motion, or reciprocating into circular motion. It consists of four links, an input link, a couple link, an output link, and a base link as shown in Fig. 4, as fixture follow the slider-crank mechanism.

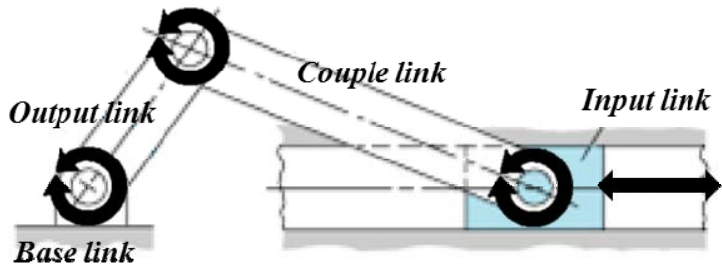

Figure 4. KINETIC MODEL OF FIXTURE FOLLOWING SLIDER-CRANK MECHANISM.

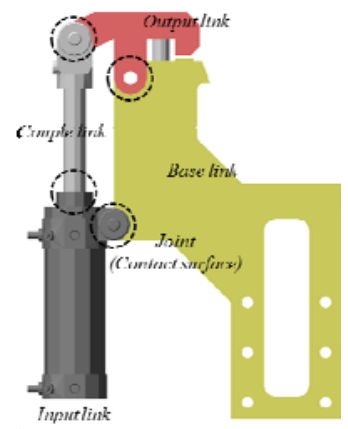

Figure 5. GEOMETRIC MODEL CONSISTING OF FOUR SUB-MODELS. 
This paper, as mentioned above, propose a procedure to extract the kinetic model from the geometric model of given fixture. It may be assumed that the geometric model of a fixture consists of four distinguishable sub-models, as shown in Fig. 5. Since a fixture follows the slider-crank mechanism, each of the four sub-models is supposed to represent one of the four links of the slider-crank mechanism (an input link, a couple link, an output link, and a base link). A type of each sub-model can be not only triangle meshes, but also primitives, such as boxes, spheres, and cylinders. The overall automated fixture modeling procedure can be described as follows.

- Step 1. The identification of the four links from the geometric model of a fixture

- Step 1-1. The extraction of common axis

- Step 1-2. The identification of joints

- Step 1-3. The matching of the link type

- Step 2. The motion planning with the identified four-axis system

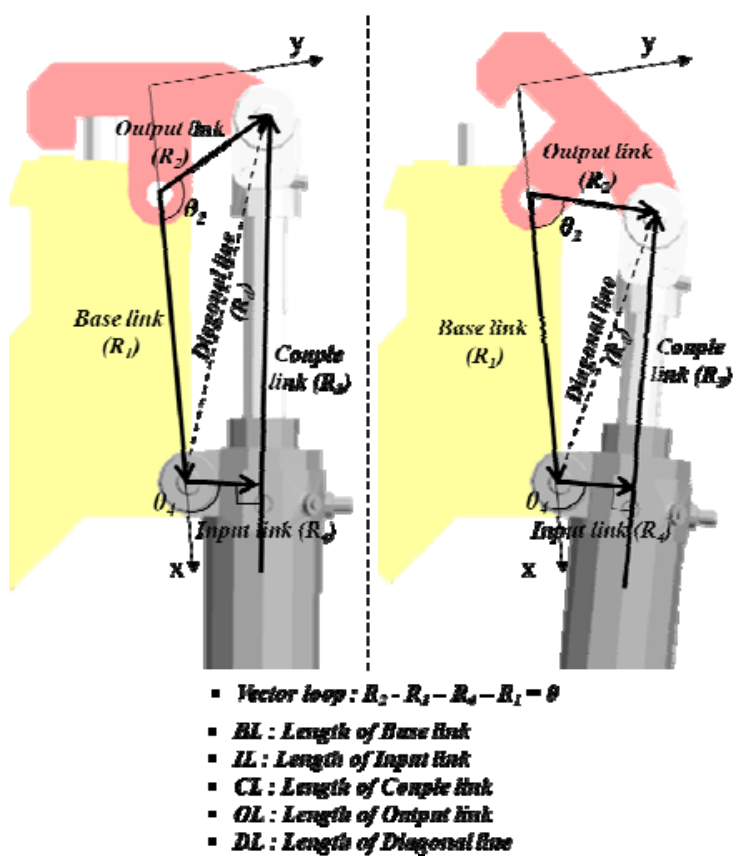

Figure 6. KINETIC LOOP OF A FIXTURE FOLLOWING INVERTED SLIDERCRANK MECHANISM.

For the first step, we find contact surface between two distinguishable sub-models to extract of common axis. Two distinguishable sub-models of a fixture are connected by a joint, such as prismatic joint, revolute joint. Since joint is a connection between two bodies that imposes constraints on their relative movement, there is contact surface between two distinguishable sub-models. We use contact surface to extract a common axis from two distinguishable sub-models. The next section addresses a detailed explanation of the algorithm.

The second step is motion planning with the identified four-axis system. As shown in Fig. 6, a jig has only two states, a 'Clamp state' and an 'Unclamp state'. It is necessary to define proper motions for the change of states. For example, an unclamping (clamping) motion changes the state of a jig from 'Clamp' to 'Unclamp' (from 'Unclamp' to 'Clamp'). It is necessary to solve the kinetic loop of a fixture to define the motions. The length of CL changes by a pneumatic actuator converting energy in the form of compressed air into linear motion, as shown in Fig. 8. The procedure to solve the kinetic loop for a given CL length is as follows.

$$
\begin{aligned}
& \text { - Step 1. } D L=\sqrt{C L^{2}+D^{2}} \\
& \text { - Step 2. } \theta_{2}=\operatorname{arcas}\left\{\frac{\sigma L^{2}+\sigma L^{2}-\sigma L^{2}}{2 \sigma L^{2} \cdot \sigma L}\right\} \\
& \text { - Step 3. } \theta_{4}=\text { formula of inverted } \operatorname{sider}-\operatorname{crank}\left(\theta_{2}\right)
\end{aligned}
$$

\section{ALGORITHM TO GENERATE A KINETIC MODEL}

This section addresses an algorithm to extract a common axis for joint of two contacted sub-models. A joint is the important element in a fixture which helps the links to travel in different kind of movement at which two or more links connect, as shown in Fig. 6. A contact is occurred by moving of links that form corresponding joint. It is very important to find a contact surface (a set of triangles) from a joint to extract a common axis. The proposed algorithm use twodimensional triangle-triangle intersection to find a contact surface. Since graphics hardware uses the triangle as its most important drawing primitive, it is only natural to perform collision detection tests on this kind of data as well. For efficient algorithm, we are concerned only about whether they intersect at all, and not interested in an exact intersection[11]. Since it is only performed on triangles, it is necessary to triangulate every shape. One of the key ideas in the proposed algorithm is to utilize the concept of the 'Gauss map'[12]. The Gauss map is the intersection of the surface normal vectors and the unit sphere, as shown in Fig. 7. Contact surface always include cylindrical features, as shown in Fig. 7. Since cylindrical feature is represented as circumference by gauss map, it is possible to extract the common axis of the corresponding joint. Fig. 8 shows common axis extracted by using the proposed algorithm.
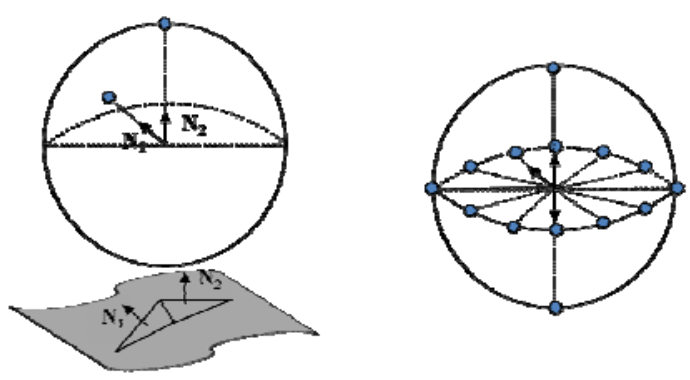

(\$) Genss mep (two polnts)

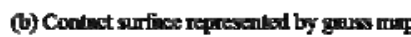

Figure 7. GAUSS MAP. 


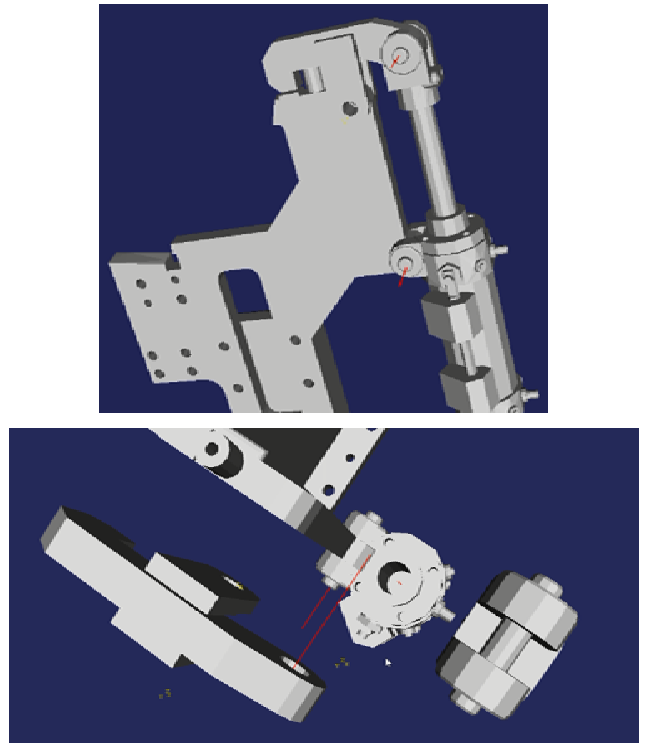

Figure 8. IMPLEMENTATION OF THE ALGORITHM TO EXTRACT A COMMON AXIS

Slider-crank mechanism, as already mentioned, consists of four bodies linked with three revolute joints and one sliding or prismatic joint. To identify each joint, we use common axis be extracted from contact surface of two submodels, as shown in Fig. 9. Although one of two sub-models connected by a prismatic joint moves along a common axis, the distance between contacted faces is not changed, unlike a revolute joint. Thus we can identify of joints through a common axis. Then, a prismatic joint be identified can be used to match a type of each link.
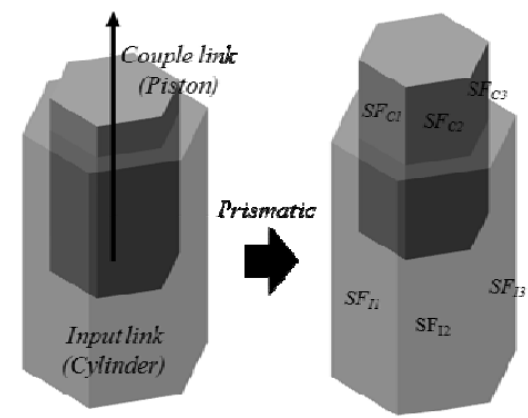

- Pair list of"prismatixicjoin

$\operatorname{Patr}\left(S F_{I D}, S F_{C 1}\right), \operatorname{Patr}\left(S F_{D D}, S F_{C 2}\right), \operatorname{Patr}\left(S F_{I 3}, S F_{C 3}\right)$

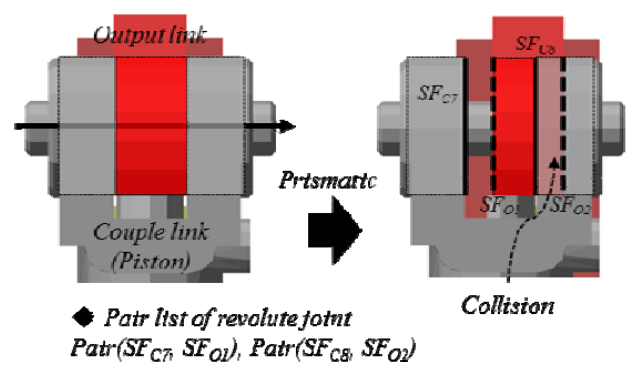

Figure 9. IDENTIFICATION OF JOINTS USING A COMMON AXIS.
In this way, it is possible to identify the prismatic axis of the slider-crank mechanism, using the concept of the moment of inertia. We propose an algorithm to identify the four links of the slider-crank mechanism by analyzing the type of a joint. We introduce a few data items, together with the basic functions to be used in the algorithm, for brevity of the explanation.

- Joint is a joint that consist of two links and one common axis. The $\mathrm{i}^{\text {th }}$ joint is denoted as joint ${ }_{i}$.

- Link is a sub-model that consist of triangles. The ith link is denoted as $\operatorname{link}_{i}$.

- Area(link) : function that returns the area of a set of triangles.

- JointType(joint) : function that returns the type of a joint, such as prismatic or revolute.

- GetLink(i) : function that returns $\operatorname{link}_{i}$.

- Four links of the slider-crank mechanism, an input link (IL), a couple link (CL), an output link (OL), and a base link (BL)

The problem of identifying the slider-crank mechanism from a joint can be described as follows:

Slider-crank mechanism identification

- Input: Four sub-models that consist of triangles

- Output: Four links of the slider-crank mechanism, IL, CL, OL, BL

// Identification of IL \& CL

For $(\mathrm{i}=0 ; \mathrm{i}<4 ; \mathrm{i}++)$

\{

If ( JointType $\left(\right.$ joint $\left._{i}\right)$ is not prismatic )

Continue;

$\operatorname{link}_{0}=$ GetLink $(0)$;

$\operatorname{link}_{1}=$ GetLink(1);

If $\left(\operatorname{Area}\left(\operatorname{link}_{0}\right)>\operatorname{Area}\left(\operatorname{link}_{1}\right)\right)$

\{

$\operatorname{link}_{0}=\mathrm{IL} ; \operatorname{link}_{1}=\mathrm{CL}$;

\}

else

\{

$\operatorname{link}_{0}=\mathrm{CL} ; \operatorname{link}_{1}=\mathrm{IL}$;

\}

\}

// Identification of OL \& BL

For $(\mathrm{i}=0 ; \mathrm{i}<4 ; \mathrm{i}++)$

\{

If $\left(\operatorname{link}_{i}==\right.$ IL or $\operatorname{link}_{i}==$ CL $)$

Continue;

If ( common axis between $\operatorname{link}_{i}$ and IL )

$\operatorname{link}_{i}=\mathrm{BL}$;

If ( common axis between link $k_{i}$ and IL )

$\operatorname{link}_{i}=\mathrm{OL}$;

\}

It is possible to extract the kinetic model from the geometric model of a fixture using the proposed algorithm. Once the kinetic model of a fixture is identified, it is possible to perform the motion planning with the slider-crank mechanism, as shown in Fig. 6. 


\section{SUMMARY}

Computer-aided fixture design has become a means of providing solutions in fixture design. Although fixtures can be designed using CAD functions, a lack of scientific tools and a systematic approach to the evaluation of design performance leads to trial-and-error strategies that result in several problems, such as the overdesign of functions, the long cycle time of fixture design. This paper proposes a procedure to extract the kinetic model from the geometric model of a fixture to reduce the modeling time and effort. Although there are various types of fixtures used in the automotive body assembly line, most of their kinetic models follow the slider-crank mechanism consist of three revolute joints and one prismatic joint. It is necessary to identify the four links of the slider-crank mechanism from the given geometric model to extract the kinetic model of a fixture. To do so, it is necessary to go through two steps; 1) Identification of the four links from the geometric model of a fixture, and 2) Motion planning with the identified four-axis system.

The focus of this paper is to extract a common axis from contact surface between distinguishable links by the concept of the 'Gauss map' that is the intersection of the surface normal vectors and the unit sphere. Contact surface always include cylindrical features, it can be represented as circumference by gauss map. It is possible to extract a common axis from a contact surface.

\section{ACKNOWLEDGMENT}

This work was supported by the Agency for Defense Development (ADD), Business for Cooperative R\&D between Industry, Academy, and Research Institute funded Korea Small and Medium Business Administration in 2013 under the Contract No. UD120035JD, C0003579. The authors wish to express sincere gratitude for the financial support.

\section{REFERENCES}

[1] Klingstam P, Gullander P. "Overview of simulation tools for computer-aided production engineering," Computers in Industry, vol. 38, Issue 2, pp. 173-186, March 1999.

[2] Ye L, Lin F, "Virtual system simulation - A step beyond the conventional simulation," 22nd Int. Conf. on Computer and Industrial Engineering, pp. 304-306, December 1997.

[3] Anglani A, Grieco A, Pacella M, Tolio T. "Object-oriented modeling and simulation of flexible manufacturing system: a rule-based procedure," Simulation Modeling Practice and Theory, vol. 10, Issues 3-4, pp. 209-234, November 2002.

[4] Asada H, By A. "Kinematic analysis of workpart fixturing for flexible assembly with automatically reconfigurable fixtures,” IEEE Journal of Robotics and Automation, RA-1(2), pp. 86-94, July 1985.

[5] Chou YC, Chandru V, Barash MM. "A mathematical approach to automatic configuration of machining fixtures: Analysis and Synthesis,” Journal of Engineering for Industry, vol. 111, Issue 4, pp. 229-306, Nevember 1989.

[6] Trappey AJC, Liu CR. "Automatic workholding verification system," Robotics and Computer-Intergrated Manufacturing, vol. 9, Issues 4-5, pp. 321-326, August-October 1992.

[7] Mervyn F, Kumar AS, Nee AYC. "Automated synthesis of modular fixture design using and evolutionary search algorithm,” International Journal of Production Research, vol. 43, Issue 23, pp.5047-5070, April 2005.

[8] Choubey AM, Prakash, Chan FTS, Riwari MK. "Solving a fixture configuration design problem using genetic algorithm with learning automata approach,” International Journal of Production Research, vol. 43, Issue 22, pp.4721-4743, March 2005.

[9] Yiming Rong, Samuel Huang, Zhikun Hou, Advanced ComputerAided Fixture design, CA:Academic Press, 2005.

[10] Minho Chang, Minsuk Ko, Sang C. Park, "Fixture modelling for an automotive assembly line,” vol. 49, No. 15, pp. 4593-4604, September 2010.

[11] Tomas Moller, “A Fast Triangle-Triangle Intersection Test,” vol. 2, Issue 2, pp. 25-30, April 1997.

[12] David A. Hoffman, Robert Osserman, The geometry of the generalized gauss map, CA:American Mathematical Society, 1980. 